$\xi_{p}$

\title{
Development of a Quantitative Assessment System for Upper Limb Motor Disorder in Occupational Therapy
}

\author{
Mohd Nor Azmi Ab Patar ${ }^{1}$, Hokyoo Lee ${ }^{2}$, Jamaluddin Mahmud ${ }^{3}$, Azim Patar ${ }^{4}$ \\ ${ }^{1,3}$ Faculty of Mechanical Engineering, Universiti Teknologi MARA, Shah Alam, Selangor Malaysia \\ ${ }^{2}$ Department of Mechanical and Control Engineering, Niigata Institute of Technology, Japan \\ ${ }^{4}$ Department of Neurosciences, School of Medical Sciences, Universiti Sains Malaysia, 16150 Kota Bharu Kelantan \\ *Corresponding authorE-mail : azmipatar@salam.uitm.edu.my
}

\begin{abstract}
Many stroke patients nowadays prefer home rehabilitation, which limits their access to proper rehabilitation equipment, treatment, or assessment by therapists. Currently, the rehabilitation programs neglect to maintain a patient's motivation in doing rehabilitation exercises. The programs also lack a monitoring system to evaluate the patients' performance quantitatively although this is the most crucial element in rehabilitation. In order to solve these problems, we have developed a novel measurement system for upper limb motor disorder rehabilitation using an optical sensor. This system consists of an optical sensor device, a personal computer and a computerized calculated program for upper limb locus position. The optical sensor detects the user's motions and calculates position and velocity. The sanding block position, velocity and the motion length of the block during the sanding task of five subjects with motor function disorder were measured. The accuracy of the trajectories was similar in all the actual measured subjects. These outcomes have a potential for the development of rehabilitation training programs and evaluation methods.
\end{abstract}

Keywords: Stroke, Hemiplegia, Upper Limb Rehabilitation, Upper Limb Motion, Measurement System, Occupational Therapy

\section{Introduction}

The development of medical technology and medical equipment benefits human health and prolongs human life. It needs to counter various problems related to aging due to the prolongation of life expectancy [1]-[4]. Thus, there is a great need for rehabilitation training due to an increase in motor disorder and cognitive impairment, resulting from cerebrovascular disease [5] that affects elderly people [6]-[8].

Previously, most of the conventional motor disorder rehabilitation, training and function recovery assessment approaches relied only on qualitative evaluation by observation. The evaluation by physicians, physiotherapists and occupational therapists was relatively subjective and different. Therefore, quantitative evaluation with objective and accurate measurement system is required [9]. The total time training with sanding movement and desk wiping motion, which is widely used in occupational therapy, is based on individual effort and the occupational therapist's subjectivity. It is difficult to judge whether a patient has reached or achieved a sufficient degree of movement to shift to the next level of training [10]. There are several devices for upper limb function training at the development stage [11][12], which are bulky and require a large space. Thus, in general a common rehabilitation center is necessary to provide such devices. However, in reality it is difficult to use it for patients [13].

Normally, occupational therapists perform the sanding movement for muscle training, and at the same time observe the amount of load and repetitive activity that occurs during the rehabilitation session [14] [15]. The repetitive activity and moving distance are used as indicators to recognize the effect of training. In addition, the desk-wiping motion is usually implemented as training to expand the range of motion of the patient's elbow and shoulder joints. In normal practice, assessment by occupational therapists is subjective in assessing the range of expansion of the range of motion. Moreover, it is not a quantitative assessment method. Therefore, there is a crucial need for the development of a measurement system for objective evaluation which defines the movable range of motion from the patient's upper limb and simultaneously tracks the movement trajectories during rehabilitation activities [16].

In this study, a device was developed which can measure the movement and does not restrict the sanding movement training and desk wiping motion training that are almost regularly practised in hospitals and rehabilitation centers. The measurement system has innovative features to measure the sanding movement speed and trajectories of each subject with upper limb motor disorder simultaneously during the training session. The obtained data were scrutinized using an objective and quantitative method to evaluate the effectiveness of the training. Furthermore, comparative study with the conventional evaluation sheet was also conducted at the same time during the training session.

\section{Materials and Methods}

\subsection{Development of the Measurement System}

Figure 1 illustrates the measurement system set up for the upper limb movement function using a developed optical sensor. This system consisted of an optical sensor attached to a sanding block, and a personal computer (PC) and integration with software specific to a measurement control program. The optical sensor's core part consisted of an LED, an optical sensor Integrated Circuit (IC) 
and software designed specifically for a measurement program. The optical sensor optimized and improved the wireless optical mouse as well as established a surface plane for the movement measurement device. Figure 2 shows the block used for the optical sensor and sanding movement training. The optical sensor was fixed to the bottom part of the sanding block, hence not restricting sanding movement training which was conducted during occupational therapy.

Once the sanding block moved, the optical sensor IC automatically captured movement data on the desk surface at a sampling frequency of $1 \mathrm{kHz}$, calculated each position, and calculated the movement volume in the direction of the $\mathrm{x}$ and $\mathrm{y}$-axis. The detailed calculation related to the position can be referred to in the report collection of the year of 2010 at the Hyogo Prefecture Rehabilitation Center (Hyogo Assist Tech) [17][18].

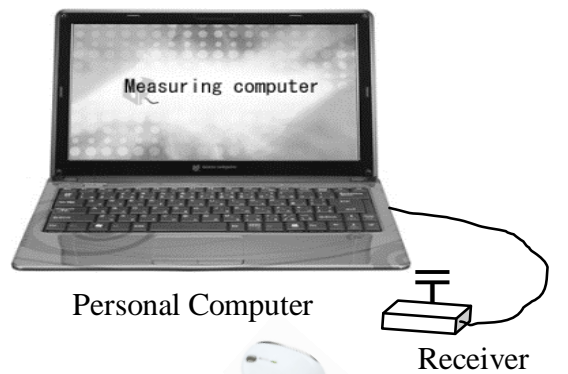

Optical sensor

Fig. 1: Overview of the optical sensor setup in the measurement system

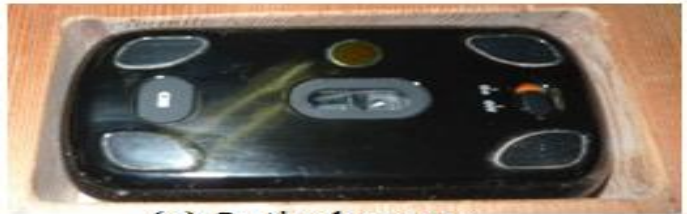

(a) Optica1 sensor
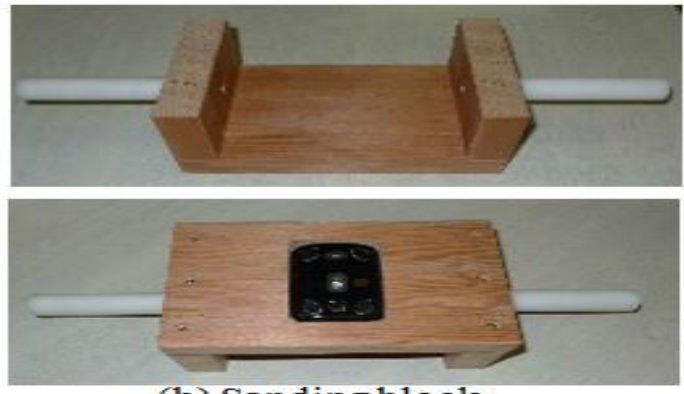

(b) Sanding block

Fig. 2: Optical sensor system and sanding block

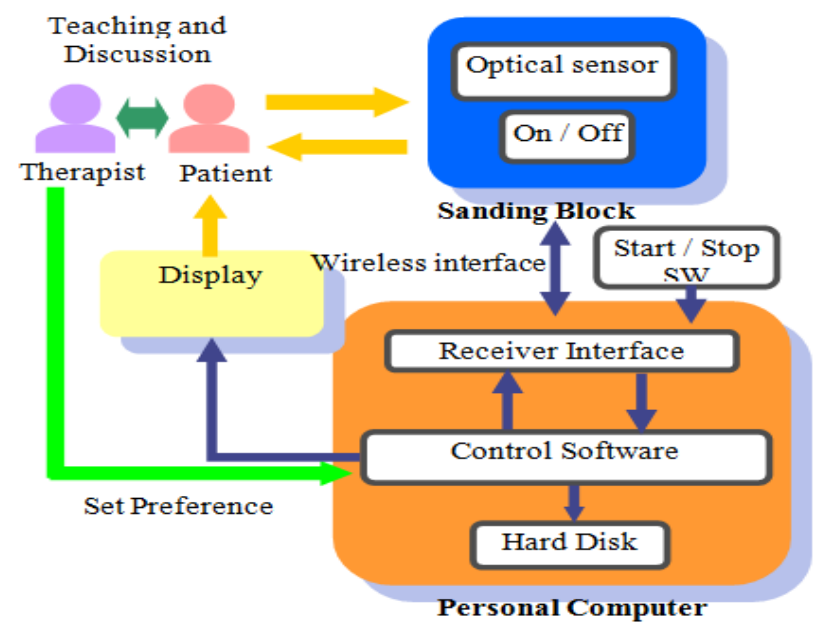

Fig. 3: Measurement device and System Control Architecture

\subsection{Motion Measurement}

Figure 3 demonstrates the overview of the operational flowchart in the block diagram which comprised the developed device and control program. In this system, an optical sensor was integrated with the sanding block body. Once the therapist or subject activated the optical sensor, the training would start after the start button was pressed. After the training finished, the therapist needed to press the stop button to stop the measurement system and the data were automatically captured and saved on the PC hard disk. The measurement system setup was mechanically simple and the operative procedure was user friendly.

\subsection{Participants}

All the subjects who participated in this experiment had motor impairment due to intracerebral hemorrhage, polyneuro pulmonary fibromatosis, cervical spinal cord injury and stroke related disease. The method of diagnosis in the study was the Manual Muscle Test (MMT) and Brunstrom stages. The upper limb motor function test indicated that the patients involved in this study could be categorised with impaired recovery stages of upper limbs in stages IV and V, hands in stages III and IV, lower limbs in stages III and IV. Table 1 shows detailed profiles of the patients. Clinical trial approval was obtained from the ethics committee of the Hyogo Prefecture Rehabilitation Center (Hyogo Assist Tech). Informed consent was also obtained before conducting a healthcare intervention on persons related experiment.

\subsection{Experiments Protocol}

During the occupational therapy session, upper limb disabled subjects did the sanding movement training with the supervision of the occupational therapist. The measurement system simultaneously measured the movement speed and displacement trajectory of each movement. Subjects sat in a wheelchair in front of the sanding block, and the technical staff then measured the height of the wheelchair and the distance from the sitting position to the operation area. The overview of the experiment setup is as illustrated in Figure 4.

During the sanding movement training, the measured data were examined according to the sanding block speed, trajectory and the repeated number of movements and distance. Since this experiment was carried out in the training program at a random speed, the achievement cycle and the number of times were different for each subject. The speed rate of each subject was calculated using equation (1).

$$
(\text { Velocity })=E\left(\sqrt{\left(\frac{d}{d t} P x(t)\right)^{2}+\left(\frac{d}{d t} P y(t)\right)^{2}}\right)_{\left[t_{1}, t_{2}\right]}
$$

Here, $\operatorname{Px}(\mathrm{t})$ is the position coordinate in the lateral direction of the sanding block, Py(t) is the coordinate in the longitudinal direction of the sanding block, $t$ is the time, $t_{l}$ is the starting time of each cycle, and $t_{2}$ is the ending time of each cycle and $E$ is the average value.

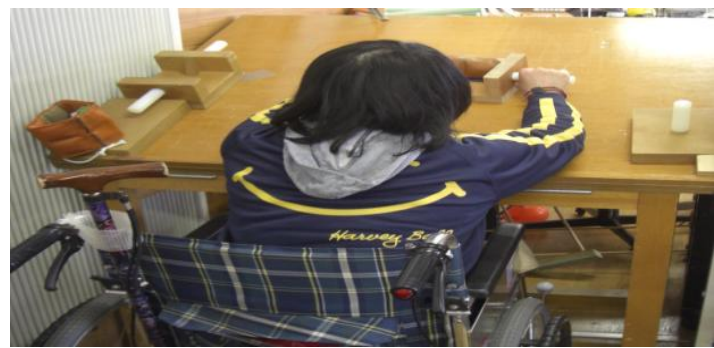

Fig. 4: Sanding movement training 


\section{Results}

The speed of the sanding movement and the position of the sanding block were automatically measured and calculated using the developed measurement system.

Table 1: Profile of five subjects

\begin{tabular}{|c|c|c|c|c|c|c|}
\hline & & Subject 1 & Subject2 & Subject 3 & Subject 4 & Subject5 \\
\hline \multicolumn{2}{|c|}{ Duration of illness (weeks) } & 14 & 28 & 61 & 26 & 14 \\
\hline \multicolumn{2}{|c|}{ Age (year) } & 60 & 37 & 51 & 42 & 28 \\
\hline \multicolumn{2}{|c|}{ Sex } & M & F & P & M & $\mathrm{P}$ \\
\hline \multicolumn{2}{|c|}{ Disorder } & $\begin{array}{c}\text { Brain } \\
\text { hemorthage }\end{array}$ & $\begin{array}{c}\text { von } \\
\text { Recklinghausen }\end{array}$ & $\begin{array}{l}\text { Spinal Cord } \\
\text { Injury C6B2 }\end{array}$ & $\begin{array}{c}\text { Brain } \\
\text { hemornhage }\end{array}$ & $\begin{array}{l}\text { Anteriorenous } \\
\text { malformation }\end{array}$ \\
\hline \multirow{3}{*}{$\begin{array}{c}\text { Brunstrom Stage } \\
\text { and manual muscle } \\
\text { testing }\end{array}$} & Hand & V & 4 & 3 & II & V \\
\hline & Upper & V & 4 & 4 & $\mathbb{I}$ & V \\
\hline & Lower & $\mathbb{V}$ & 3 & 0 & $\mathbb{V}$ & V \\
\hline
\end{tabular}

Figure 5 (a) shows the motion trajectory when subjects performed the sanding movements. From the trajectory, we observed the position of the sanding block during the training session and recognized that the repeat of extension and flexion movement region was more on the right side if compared to the original position or the center of the movement region. The behavior shown in the trajectory graph indicated that the subject has a disorder of the right hemiplegia. In the case of the right hemiplegia, the cooperative movement of both hands performed during the extension and flexion movement indicated that the movement was accomplished by the left hand rather than the right hand. Moreover, it was realized that the moving distance was not constant while each extension and flexion was executed.

From the operation cycle, it was observed that the extension and flexion movement was repeated two to three times from the start of operation. It was presumed that the two to three times is a good margin execution of the extension and flexion movement. Furthermore, the trajectory profile demonstrated that the sanding block shifted to the right, yet the lateral deviation was in the range of about $100 \mathrm{~mm}$. In Figure 5 (b), the horizontal axis shows time and the vertical axis shows the position of the sanding block during the extension and flexion movement of the training session. The working distance of the extension and flexion movement was short as it was in a duration of less than 30 seconds. In the training session of 1 minute, extension and flexion movement achieved seven times each

Figure 6 shows the operation speed during the extension and flexion movement of the training session. Both the extension and flexion movements indicated the highest speed of about $200 \mathrm{~mm} / \mathrm{sec}$, which confirmed that the training session was conducted at a constant speed.

When compared with the trajectory at around 30 seconds, the speed for a short working distance was less than $200 \mathrm{~mm} / \mathrm{sec}$ for both extension and flexion movements. It was observed at the trajectory that when the operation speed after 40 seconds was increasing, the operating distance was also relatively longer.

Figure 7 illustrates the average value of the extension and flexion movement speed when five subjects completed the sanding movement training session. On comparing the extension motion speed $(97.1 \mathrm{~mm} / \mathrm{sec})$ and the flexion motion speed $(86.3 \mathrm{~mm} /$ sec), it clearly indicated that the extension motion was faster than the flexion motion.

Table 2 shows the average value of the operating speed for 5 recruited subjects, and Figure 8 shows the graph of the average operating speed and average working distance during the sanding block movement training. The average working distance for the duration of the one-minute training session was $5.3 \pm 0.4$ metre while the average operating speed was $91.7 \pm 6.5 \mathrm{~mm} / \mathrm{sec}$.
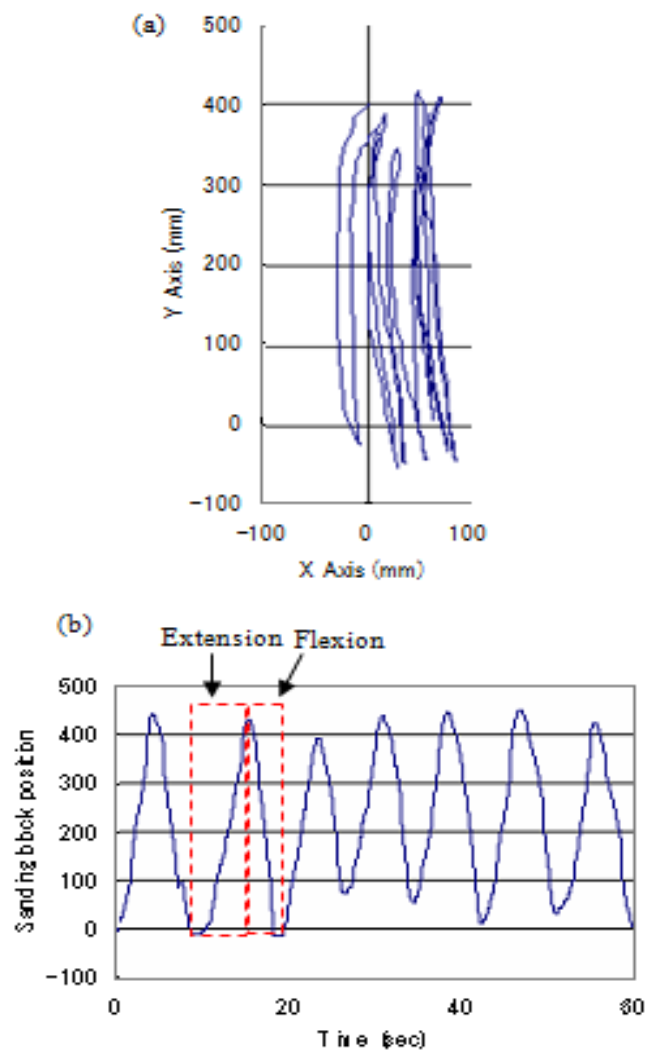

Fig. 5: Typical profiles of the sanding block position trajectory (a) and real-time position (b)

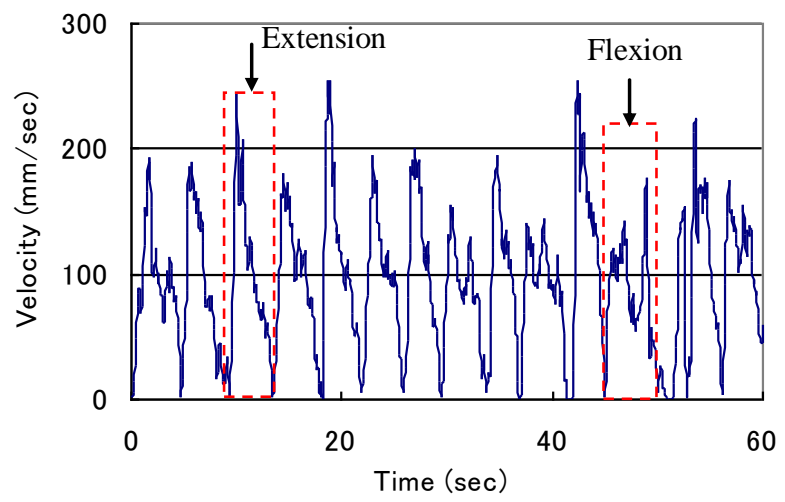

Fig. 6: Operating speed during the extension and flexion movement of training session

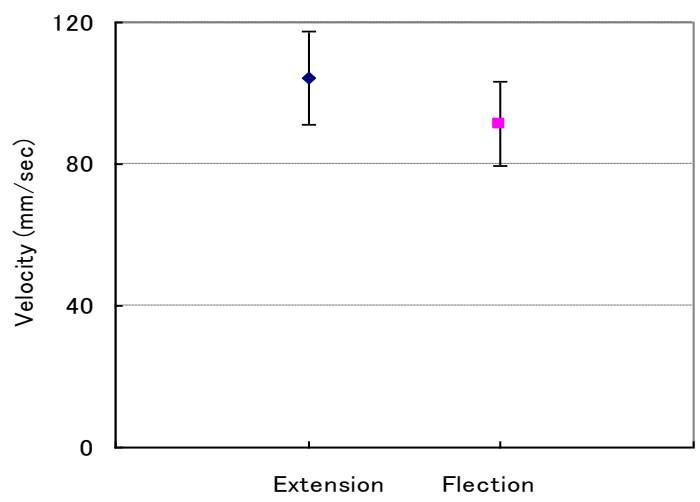

Fig. 7: Experimental results of the velocity of extension and flexion movement of 5 subjects during the sanding movement training session 
Table 2: Summary of the length and velocity of extension and flexion movement during the training session

\begin{tabular}{|c|c|}
\hline \multicolumn{2}{|c|}{ Training Session } \\
\hline Velocity of Extension $(\mathrm{mm} / \mathrm{sec})$ & $97.1 \pm 13.1$ \\
\hline Velocity of Flexion $(\mathrm{mm} / \mathrm{sec})$ & $86.3 \pm 11.7$ \\
\hline Average Velocity $(\mathrm{mm} / \mathrm{sec})$ & $91.7 \pm 6.5$ \\
\hline Length $(\mathrm{m})$ & $5.3 \pm 0.4$ \\
\hline
\end{tabular}

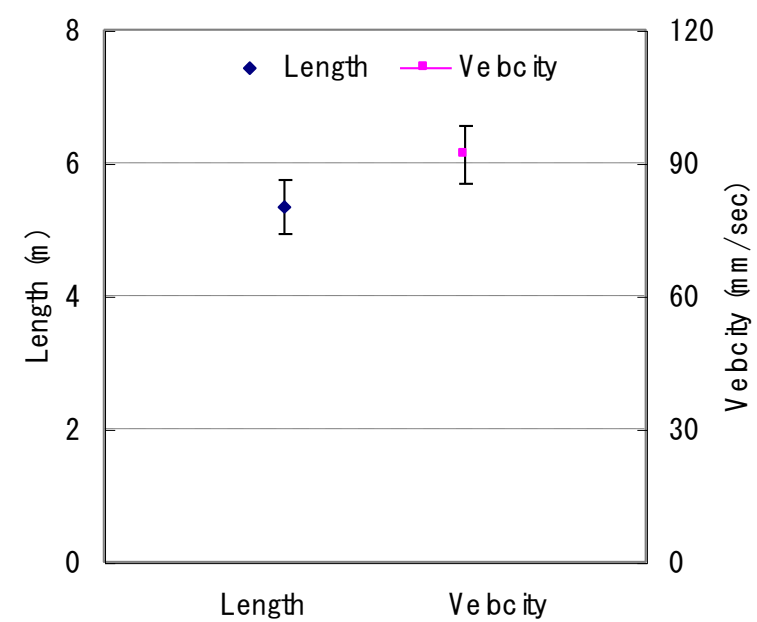

Fig. 8: Average velocity and operating distance during the sanding block movement training

\section{Discussion}

All recruited subjects were went through the measurement procedure without disturbing the sanding movement training that is normally practised in occupational therapy. There were five subjects recruited in this experiment. It was impossible to perform statistical analysis because of the training times and the different level of disabilities due to a variation of diseases after the onset of illness. In comparing the method used by measuring the repeated number of movements by a medical institution and the subjective judgement by physicians and physiotherapists, it was clear that the measurement system used was a more objective and quantitative evaluation method.

According to Lee et al., the upper limb motor function rehabilitation system can be evaluated by the parameters such as operating speed, working distance, error and other parameters during the movement of the training to recognize the behavior of the upper limb movement function [19]. Furthermore, even in clinical practice, especially to assess the patients with motor function disorder, the working distance and movement pattern is studied to observe the improvement of motor function recovery. The inspection procedure not only considers one aspect or point of view but the standard protocol of the evaluation also considers multidimensional aspects such as the progress of the motor function recovery, the mechanism of recovery, the body change to the paralyzed side of the patients and other aspects related to the patient's condition which are quantitatively analyzed [20]-[22]. Therefore, the evaluation method of the sanding movement training assessed in our novel measurement system suggests that obtaining motion distance and motion speed, which objectively evaluate the exercise capacity and the range of motion during the training session is effective.

\section{Conclusion}

In this study, a novel measurement system was developed, which has the ability to measure the amount of training when targeted patients performed the sanding movement, in order to assess upper limb motor disorder in occupational therapy. The movement speed and trajectory of the sanding movement of five recruited subjects with upper limb motor disorder were measured. It was clearly indicated that the measurement system could assess the behaviour of the patients without interrupting the movement of the five recruited subjects during the training session. The outcomes achieved from the training session are briefly summarized and discussed below.

Firstly, our system has features to demonstrate the movement trajectory in the sanding movement training. Thus, it is easy to identify the motion characteristics of every recruited subject especially during the extension and flexion movement. A simple, easy and quantitative approach to evaluate the degree of disability was implemented. It was also discovered that the extension motion was faster than the flexion motion for all recruited subjects during the sanding movement training.

Our system was able to measure the operating speed and working distance and was a beneficial approach to diagnose the recovery stages of the motor function. The measurement of the training activity and the data evaluated by the therapist improved the accessibility of the evaluation.

From this study, it was clearly revealed that our system is effective for the quantitative assessment of the degree of training at the clinical site.

In the future, a multifaceted examination is necessary, such as conducting clinical trials on a larger number of recruited subjects and generalizing the results before getting the feedback from the clinical site for our measurement device system.

\section{Acknowledgement}

The authors would like to thank all recruited subjects and the Independent Living Training Center for Disabled Persons section at the Hyogo Prefectural General Rehabilitation Center especially Mr. Honda, as an occupational therapist supporting staff during the training session.

\section{References}

[1] E. Topinková, “Aging, disability and frailty," Annals of Nutrition and Metabolism. 2008.

[2] M. Van den Akker, F. Buntix, J. F. M. Metsemakers, S. Roos, and J A. Knottnerus, "Multimorbidity in general practice: Prevalence, incidence, and determinants of co-occurring chronic and recurrent diseases," Journal of Clinical Epidemiology, 1998.

[3] K. Barnett et al., "Epidemiology of multimorbidity and implications for health care, research, and medical education: a cross-sectional study.," Lancet (London, England), 2012.

[4] J. L. Wolff, B. Starfield, and G. Anderson, "Prevalence, expenditures, and complications of multiple chronic conditions in the elderly," Archives of Internal Medicine, 2002.

[5] National Instiute of Neurological Disorders and Stroke, "Classification of cerebrovascular diseases III.," 1990.

[6] M. M. Sohlberg and C. A. Mateer, "Effectiveness of an attentiontraining program," Journal of Clinical and Experimental Neuropsychology, 1987.

[7] J. A. Edmans, J. Webster, and N. B. Lincoln, "A comparison of two approaches in the treatment of perceptual problems after stroke," Clinical Rehabilitation, 2000.

[8] L. T. Carter, B. E. Howard, and W. A. O'Neil, "Effectiveness of cognitive skill remediation in acute stroke patients.," The American journal of occupational therapy: official publication of the American Occupational Therapy Association, 1983.

[9] C. T. Debert, T. M. Herter, S. H. Scott, and S. Dukelow, "Robotic assessment of sensorimotor deficits after traumatic brain injury," Journal of Neurologic Physical Therapy, 2012.

[10] C. Counsell, M. Dennis, and M. McDowall, "Predicting functional outcome in acute stroke: Comparison of a simple six variable model with other predictive systems and informal clinical prediction," Journal of Neurology, Neurosurgery and Psychiatry, 2004.

[11] H. Igo Krebs, N. Hogan, M. L. Aisen, and B. T. Volpe, "Robotaided neurorehabilitation," IEEE Transactions on Rehabilitation Engineering, 1998

[12] K. Koyanagi, F. Furusho, U. Ryu, and A. Inoue, "Rehabilitation 
system with 3-D exercise machine for upper limb," in IEEE/ASME International Conference on Advanced Intelligent Mechatronics, AIM, 2003.

[13] Y. Morita, R. Tanioku, M. Uchida, H. Ukai, and N. Matsui, "Study on quantitative evaluation methods of therapeutic effects of sanding training," 2008 International Conference on Control, Automation and Systems, pp. 913-918, 2008.

[14] M. OOGIRI, H. YUMINAGA, N. KIDA, and H. HAMADA, "Impression Evaluation and Movement Evaluation in Gait Observation of Rehabilitation Patient," Transactions of Japan Society of Kansei Engineering, vol. 16, no. 1, pp. 181-187, 2017.

[15] H. YOSHIYUKI et al., “Assessment of Independent Walking by Stroke Patients: Effects of an Assessment Sheet Used in Convalescence Rehabilitation Ward," Rigakuryoho Kagaku, pp. 635-639, 2016.

[16] H. K. Lee, Y. T. Kim, Y. Takahashi, T. Miyoshi, K. Suzuki, and T. Komeda, "Basic experiment on rehabilitation of upper-limb motor function using haptic-device system," Transactions of the Korean Society of Mechanical Engineers, A, 2011.

[17] H. LEE et al., "2P2-B10 Development of measuring equipment for upper limb motor function rehabilitation using optical sensor(Rehabilitation Robotics and Mechatronics)," The Proceedings of JSME annual Conference on Robotics and Mechatronics (Robomec), vol. 2011, p. _2P2-B10_1-_2P2-B10_4, 2011.

[18] T. Lee et al., "Basic experiments of upper limb rehabilitation using haptic device system," 9th International Conference on Rehabilitation Robotics, 2005. ICORR 2005., pp. 444-447, 2005.

[19] M. N. A. A. Patar, T. Komeda, L. C. Yee, and J. Mahmud, "Modelbased systems engineering of a hand rehabilitation device," Jurnal Teknologi, vol. 76, no. 4, pp. 101-106, 2015.

[20] M. N. A. B. A. Patar, T. Komeda, J. Mahmud, and C. Y. Low, "Model Based Design of Finger Exoskeleton for Post Stroke Rehabilitation Using a Slotted Link Cam with Lead Screw Mechanism," in Industrial Engineering, Management Science and Applications 2015, Berlin, Heidelberg: Springer Berlin Heidelberg, 2015, pp. 95-103.

[21] T. Miyoshi, Y. Takahashi, H. Lee, and T. Terada, Haptic Device System For Upper Limb Motor Impairment Patients : Developing And Handling In Healthy Subjects, no. August. 2007.

[22] T. Miyoshi, Y. Takahashi, H. Lee, T. Suzuki, and T. Komeda, "Upper limb neurorehabilitation in patients with stroke using haptic device system: Reciprocal bi-articular muscle activities reflect as a result of improved circle-drawing smoothness," Disability and Rehabilitation: Assistive Technology, vol. 5, no. 5, pp. 370-375, 2010 . 